\title{
Improving Spinal Cord Stimulators' Outcomes: A literature Review
}

\author{
Gina Kelly-Vasquez ${ }^{1}$
}

\section{Abstract}

Spinal pain is debilitating, inhibits the quality of life, requires many interventions that include non-pharmacological, pharmacological therapies that are costly to both patients and insurance companies. With the advent of updated chronic pain treatment technology, it is essential to accurately assess risks versus benefits and many varying contributing factors in treatment candidacy.

The purpose of the exploratory research is to describe current pain neuromodulation technology that includes spinal cord stimulators (SCS) and dorsal root ganglion (DRG) technology. The employed research methods are secondary research analysis comprised quantitative and qualitative data.

The research revealed that when individuals undergoing the SCS trial period, if they experienced 4.6 out of ten pains, there is a 50\% probability of permanent implantation success with a sensitivity of 97.14 and specificity of $44.44 \%$. An individual who underwent DRG implantation within 1-year, complex regional pain, and failed back syndrome was reduced by $-56.7 \%$, back pain was $-46.71 \%$, and foot pain $-72.97 \%$. In one reviewed study conducted from 1997 to 2014, 73\% of permanent devices were removed due to inadequate pain control. In another reviewed research conducted from 2013 to 2017, 22\% reported complications causing a need for device removal.

It is concluded that although there is a trial period for the candidacy of permanent placement of SCS or DRG, there is a high incidence of permanent device explanation. There is a need for more exploratory research on causative variables that result in permanent neuromodulation device removal. Thorough documenting of baseline pain, along with thorough psychological clearance, can aid in providing enhanced outcomes after permanent implantation.

Keywords: Spinal cord stimulators; Implants; Generators; Explantation; Revisions.

\section{Introduction}

Spinal pain is subjective, and with the aid of imaging, surgeons can determine causative factors. For some individuals minimizing pain can be accomplished with high-quality surgery that can also contribute to reducing health-care costs and advance medical research. Post-operative complications can occur with any given procedure, and many can be managed. Conductive thorough pre-operative care, which includes trials, can help in providing enhanced valid outcome measures that are reliable, and clinically significant for best practice.

Chronic pain is complex and challenging, and it is estimated that one in five adults experience chronic pain [1]. The

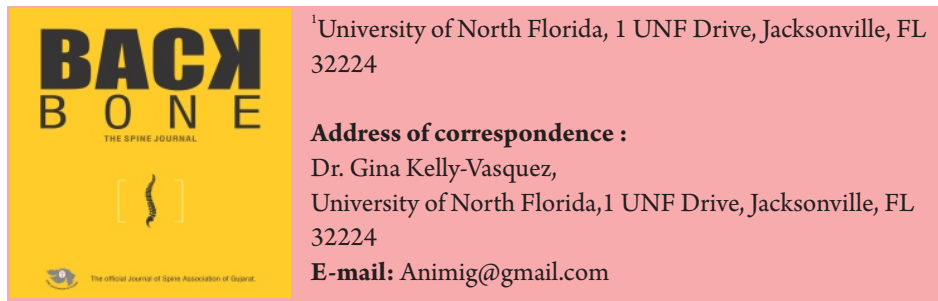

complexity of chronic pain requires a multidisciplinary approach that includes pharmacological, physical, psychological, and surgical interventions. Pharmacological interventions may function short-term, but when used for long-term use, data are limited in supporting efficacy in reducing pain [1]. Chronic spinal pain can be the result of spinal cord injuries; neuropathic and radicular pain includes failed back surgery syndrome (FBSS) and complex regional pain syndrome (CRPS). In descending order, the mostreported pain etiologies of pain are radiculopathy neuropathic pain, degenerative disc disease, and FBSS [1]. The mostreported pain locations are neck pain and the upper limb pain [1].

The prevalence of chronic pain increases with age, and its incidence is expected to continue to rise [1]. Chronic pain is estimated to cost beyond $\$ 100$ billion, which includes associated costs of chronic opioid therapy [2]. The costs include the effects of chronic opioid therapy, which involve substance abuse, gastrointestinal side effects, respiratory commercial use, distribution, and reproduction in any medium, provided the original work is properly cited. 
depression, overdose, and mortality [2]. Costs of modern treatment modalities as that of spinal cord stimulators (SCS) come with many hopes in lowering such costs but are under much scrutiny by third-party payers. Consequently, ensuring efficiency with clinical efficacy must be factored when recommending patients to undergo surgery as it can hinder future reimbursement when patients report minimal to no improvement; insurance companies can deem surgery as ineffective, not durable or reliable. Because of SCSs posing benefits to improve chronic pain and lowering costs long-term, it is crucial to consider causative factors between trial period and permanent placement by having more thorough documentation of patients' baseline, trial, and postimplantation pain evaluation.

\section{Discussion}

Neuromodulation arose as an effort to pain relief for patients with chronic pain. With the advance of knowledge of pain receptors and technology, SCS and now Dorsal Root Ganglion (DRG) stimulation have become adjunct therapies.

SCS was the first stimulation intervention with aims in reducing complex pain syndromes, which also include individuals who failed to reduce pain after back surgeries. The way SCS functions are that it inhibits the neural activity of nociception-transmitting fibers in the spinal cord's dorsal horn [3]. As of 2018, it is estimated that 50,000 SCSs are implanted annually [4].

The indications approved by the FDA are chronic neuropathic pain of the trunk and limbs, FBSS, and CRPS that include reflex sympathetic dystrophy and causalgia [5]. Pre-operative workup consists of a complete blood count, coagulation panel, platelet function test, anticoagulant clearance, and free from infections [6]. Post-surgery follow-up is conducted a month after, where the incisions are assessed, and sutures are removed if applicable [7]. Managing the stimulator is done with a pain specialist or the manufacturer's representative. The most common risk or complications occur within the 1st postoperative months that include lead migration, and lead fracture requiring re-positioning. According to Haider et al. [5], since complications are related to hardware, they are not permanent sequelae.

DRG is the newest form of decreasing nociceptive and neuropathic pain in focal areas that SCS cannot reach. Focal areas include the groin, the trunk, a hand, a foot, and sacral area. The pulse or frequency generator includes wiring, have chargeable or non-chargeable units, which are implanted percutaneously and a laminectomy [8]. Many dermatomes are stimulated, where the individual may experience mild or minimal paresthesia with the individual controlling it by turning intensity down with remote control.

It is essential to highlight the contraindications to spinal nerve stimulation include infections, cardiac pacemakers, defibrillators, as well as blood clotting conditions [9]. Other reasons for contraindication include negative clearance by primary care and pertinent allied health providers.

Orhurhu et al. [3] conducted a retrospective study where 88 patients were identified during the SCS trial period and followed up from 2015 to 2018 . The objective of the study was to determine the correlation between SCS trial pain scores, and those after the permanent SCS implantation. The study found that if an individual experienced a 4.6 out ten levels of pain during the trial, there was a $50 \%$ probability of permanent implantation success with a sensitivity of 97.14 , and specificity of $44.44 \%$ [3]. One of the most prominent findings was that women tend to have a higher probability (13\%) of success. Although this study had set parameters for the study, it is recommended to have further research to determine what other factors may yield positive outcomes.

Compared to SCS, DRG studies have revealed that year postimplantation failed back syndrome, and complex regional pain was reduced from 77.6 to $33.6(-56.7 \%)$ [10]. Back pain was decreased from 74.5 to $39.7(-46.71 \%)$ and with the highest reduction in foot pain from 81.4 to $22.0(-72.97 \%)$ [10].

\section{SCS Manufacturers and Costs}

Manufacturers of SCSs include Medtronic Inc, Abbott, Boston Scientific, and Nevro Corp [9]. The technology within the SCSs continues to evolve. Some models are smaller, chargeable, and non-chargeable. They can deliver higher dose therapies and can be compatible with magnetic resonance imaging (MRI).

Current procedure terminology codes for neuromodulators include procedures involving the implants, generators, removals, and revisions. There are also the Healthcare Common Procedure Coding System codes that bill for leads, receivers, and other pertinent durable medical equipment [11]. According to Woolstenhulme (2019), the base cost for the trial period of the SCS is $\$ 6790.33$, and its service code is 63650 . The base cost for removing the electrode is $\$ 2980.80$, and its service code is 63661 [11]. The service code for the permanent implantation is 65350 , with a value of $\$ 6790.33$, and code 63685 is the insertion of the pulse generator with a base code of $\$ 1725.64$ [11]. The combined total from the trial phase to permanent implantation of stimulators is $\$ 18287.1$.

\section{Considerations/Recommendations}

Although spinal cord stimulation implantation requires trial periods before permanent placement, there is a high incidence of patients requesting hardware removal. Some of the reasons for removal include patients' perception of inadequate pain control, discomfort with hardware, and among many others. An institutional review board was conducted within a hospital 
setting from 1997 to 2014, a total of 946 charts were reviewed, and a total of 165 patients were targeted for the final review. Approximately $66 \%$ were females, with the median time with implantation of 665 days [12]. The highest percentage, which was $73 \%$ of those individuals who had stimulator removed, reported inadequate pain control. The second highest reported reason for removal at $22 \%$ was discomfort at the site of electrode placement, $10 \%$ decided to have the removal due to not wanting to undergo MRI imaging, $9 \%$ decided explantation due to dysesthesias, and wound dehiscence, and $2 \%$ experienced weakness. In comparison, $1 \%$ experienced muscle spasms [12]. To further evaluate the highest group that reported inadequate pain control, the review board found that $39.1 \%$ lost pain control over time [12].

Another institutional review board conducted a retrospective chart review of 100 patients who had surgery from 2013 to 2017. In this study, the focus was to identify pain relief at trial and pain relief up to 6 weeks post permanent implantation. If individuals reported more than $50 \%$ pain relief during the trial period, they were candidates for permanent placement. Out of those individuals who had a permanent placement, $22 \%$ reported complications, and 15 patients experienced prolonged pain at the incision site, while five patients developed infection symptoms [8].

SCSs are invaluable adjunct therapies to help individuals to cope with radiculopathy and lumbar pain. However, what helps one individual will not help all, as in SCS patients. Not everyone experiences total pain relief or permanent resolution. An essential factor is that of expectations versus outcomes based on the trial period. There could also be the correlation of patients reporting higher levels of pain after permanent implantation as surgery is more invasive than during the trial periods. Therefore, it is recommended that more exploratory research is done on what are the causative variables that result in permanent neuromodulation device removal. Thorough and proper documentation of patients' expectations after implantation, pain level, symptoms, and characterization of baseline pain are the start in exploratory data gathering. Having a thorough pre-operative workup that includes psychological clearance and medical history can also determine long-term benefits. During the trial spinal cord generator period, comprehensive documentation of pain level and characteristics, frequencies employed, pain medication dosing or titrating down, as well as meeting the lowest acceptable pain to conduct activities of daily living. Having the individual identify the lowest level of tolerable pain during the trial period is essential as it will elude to coping with minimal pain during permanent implantation. Knowing the different pain dimensions for each patient can differentiate between postoperation pain and long-term results of the permanent spinal cord generator.

\section{Conclusion}

Before considering a patient as an adequate candidate for permanent SCS, there are several factors to consider. The individuals must undergo a thorough psychological clearance that will add toward the patient understanding there will be a decrease and ultimate discontinuation of narcotic medications. Both the surgeon and the patient have a clear understanding of what the patients' hope to gain from undergoing surgery. Other factors to consider in the pre-operative workup include age, history, manufacturer as part of the education, and baseline information gathering. During the trial period, information is continual and assessed daily, which provides further details on the lowest pain attained, thus possible pain level at permanent placement. At the time of permanent placement, the provider must distinguish post-operation pain from ineffective surgery outcomes. It is crucial for all parties involved to understand that improved outcomes may occur upon several weeks. Subsequently, results are measured individually with the aid of imaging to determine proper placement and lead stability.

\section{References}

1. Sayed D, Kallewaard JW, Rotte A, Jameson J, Caraway D. Pain relief and improvement in quality of life with $10 \mathrm{kHz}$ SCS therapy: Summary of clinical evidence. CNS Neurosci Ther 2020;26:403-15.

2. Hoelscher C, Riley J, Sharan A, Wu C. Cost-effectiveness data regarding spinal cord stimulation for low back pain. Spine (PhilaPa 1976) 2017;42:S72-9.

3. Orhurhu V, Chu R, Orhurhu MS, Odonkor CA. Association between pain scores and successful spinal cord stimulator implantation. Neuromodulation 2019;23:660-6.

4. Sdrulla AD, Guan Y, Raja SN. Spinal cord stimulation: Clinical efficacy and potentialmechanisms. Pain Pract2018;18:1048-67.

5. Haider SA, Wilock ME, Pilitsis JG. Complication avoidance and revision surgery.
In: Mammis A, editor. Spinal Cord Stimulation Principles and Practice. Hauppauge: Nova Science Publishers Inc.; 2016. p. 95-110. Available from: http://www.search.ebscohost.com.dax.lib.unf.edu/login.aspx?direct=trued $d b=$ nlebke' an $=1164744 \mathrm{e}^{\prime}$ 'site $=$ eds-live ${ }^{\prime}$ 'scope $=$ site.

6. Kaufman A, Shah R. Indications and patient selection. In: Mammis A, editor. Spinal Cord Stimulation Principles and Practice.

Hauppauge: Nova Science Publishers Inc.; 2016. p. 8-12. Available from: http://www.search.ebscohost.com.dax.lib.unf.edu/login.aspx?direct=trued db= nlebke' an $=1164744$ 'site $=$ eds-livee'scope $=$ site.

7. Anderson B, Cockroft KM. Spinal Cord Stimulation. Rolling Meadows: American Association of Neurological Surgeons; 2020. Available from: 
https://www.aans.org/patients/neurosurgical-conditions-andtreatments/spinal-cord-stimulation. [Lastaccessed on 2020 Apr 05].

8. Malige A, Sokunbi G. Spinal cord stimulators: A comparison of the trial period versus permanent outcomes. Spine (Phila Pa 1976) 2019;44:E687-92.

9. Springston GM. Spinal cord stimulators-the basics and the buzz. J Nurse Life Care Plan 2019;19:29-35. Availablefrom:

http://www.search.ebscohost.com.dax.lib.unf.edu/login.aspx?direct=truew $d b=r z h$ \& an $=138528281$ \& site $=$ eds-live ${ }^{\prime}$ scope $=$ site.

10. Verrills P, Sinclair C, Barnard A. A review of spinal cord stimulation systems for chronic pain.JPain Res 2016;9:481-92.

11. Woolstenhulme C. Spinal cord stimulator coding. J Nurse Life Care Plan 2019;19:36-7. Availablefrom:

http://www.search.ebscohost.com.dax.lib.unf.edu/login.aspx?direct=trued $d b=r z h$

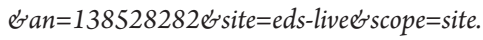

12. Dupre DA, Tomycz N, Whiting D, Oh M. Spinal cord stimulator explantation: Motives for removal of surgically placed paddle systems. Pain Pract 2018;18:500-4.
Conflict of Interest: NIL

Source of Support: NIL

\section{How to Cite this Article}

Vasquez GK| Improving Spinal Cord Stimulators' Outcomes: A literature Review| Back Bone: The Spine Journal | October 2020-March 2021; 1(1):33-36. 